\title{
SOME REMARKS ON CATEGORY IN TOPOLOGICAL SPACES
}

\author{
ZBIGNIEW PIOTROWSKI AND ANDRZEJ SZYMAŃSKI
}

ABSTRACT. We prove some results concerning the covering, additivity, and the uniform numbers for general topological spaces.

For any dense-in-itself $T_{1}$ topological space $X$ let us put $\operatorname{cov}(X)=\inf \{|R|: R$ is a family of nowhere dense subsets of $X$ covering $X\}$. Then $\operatorname{cov}(X)$ is always an infinite cardinal that does not exceed $|X|$, the cardinality of $X$. If $\operatorname{cov}(X)$ is an uncountable cardinal, then $X$ is second category, and if $\operatorname{cov}(U)$ is uncountable for every nonempty open subset of $X$, then $X$ is a Baire space.

Let us put $\operatorname{cov}^{2}(X)=\inf \{\operatorname{cov}(F): F$ is a closed, nonempty, dense-in-itself subspace of $X$ \}. If $\operatorname{cov}^{2}(X)$ is an uncountable cardinal, then $X$ is usually called a totally nonmeager space [1] (sometimes it is also called a totally inexhaustible space [8]).

In [8], the behavior of totally nonmeager spaces under feebly continuous and feebly open mappings have been considered (see also [2]). Let us recall [4] that a mapping $f$ of a space $X$ onto a space $Y$ is feebly continuous if int $f^{-1}(V) \neq \varnothing$ whenever $V$ is nonempty and open in $Y$, and $f$ is feebly open if int $f(U) \neq \varnothing$. whenever $U$ is nonempty and open in $X$. T. Neubrunn [9] conjectured that

(*) If $f$ is a one-to-one feebly continuous and feebly open mapping of a regular space $X$ onto a totally nonmeager space $Y$, then $X$ is a Baire space.

We shall show that this conjecture is true even without appealing to any additional separation axioms. For this purpose we prove the following general result.

THEOREM 1. Let $f$ be a one-to-one feebly continuous and feebly open mapping of a space $X$ onto a space $Y$. If $U$ is a nonempty open subset of $X$, then $\operatorname{cov}^{2}(Y) \leq$ $\operatorname{cov}(U)$.

ProOF. Suppose that $U$ is a nonempty open subset of $X, R$ is a family of nowhere dense subsets of $U$ that covers $U$, and $|R|=\operatorname{cov}(U)$. Let us put $W=$ int cl int $f(X-\operatorname{cl} U)$. Then $W$ is an open subset of $Y$ that is disjoint with int $f(U), f$ being one-to-one. We shall show that $\operatorname{cov}(F) \leq \operatorname{cov}(U)$, where $F=Y-W$. The closed set $F$ can be decomposed into two disjoint parts: $F_{1}=\operatorname{int} f(U)$ and $F_{2}=F-\operatorname{int} f(U)$. Part $F_{2}$ is a closed subset of the closed set $F$. Let us check that it is also nowhere dense in $F$. So let $V$ be an open subset of $Y$ such that $F_{2} \cap V \neq \varnothing$. Hence $V-W \neq \varnothing$ and, because $V$ is open $G=V-\operatorname{cl}$ int $f(X-\operatorname{cl} U) \neq \varnothing$. Because $f$ is feebly continuous, int $f^{-1}(G) \neq 0$. This set has to be disjoint with $X-\operatorname{cl} U$; otherwise $G \cap f(X-\operatorname{cl} U) \neq \varnothing(f$ being feebly open $)$, which is impossible. Hence int $f^{-1}(G) \subset \operatorname{cl} U$, and therefore int $f^{-1}(G) \cap U \neq \varnothing$. Because $f$ is feebly open,

Received by the editors January 23, 1986 and, in revised form, May 26, 1986.

1980 Mathematics Subject Classification (1985 Revision). Primary 54A25; Secondary 54E52.

This paper was completed while the second named author was a Visiting Professor at Youngstown State University. 
$G \cap \operatorname{int} f(U) \neq \varnothing$. Since $G \subset V, V \cap \operatorname{int} f(U) \neq \varnothing$ which shows that $F_{2}$ is nowhere dense in $F$.

To prove our theorem now, it is enough to show that int $f(U)$ can be covered by $\operatorname{cov}(U)$ or a less nowhere dense subset of $F$. We will have this if we show that for any $E \in R, F_{E}=\operatorname{int} f(U) \cap f(E)$ is a nowhere dense subset of $F$. To see this we argue in the following way. The image of a nowhere dense subset of $X$ under a one-to-one feebly open and feebly continuous mapping is again a nowhere dense subset of $Y$. Nowhere dense subsets of an open set of a space are exactly those sets which are subsets of the open set and nowhere dense in the space. Therefore each $F_{E}$ is a nowhere dense subset of int $f(U)$, being the intersection of a nowhere dense set in the space $Y$ with an open subset of the space $Y$. Because int $f(U)$ is contained in $F$, those sets are also nowhere dense in $F$.

As we know, $\operatorname{cov}^{2}(Y)>\omega$ means the same as $Y$ being totally nonmeager, and $\operatorname{cov}(U)>\omega$ for each nonempty open subset of $X$ means the same as $X$ being a Baire space, so $(*)$ follows immediately from Theorem 1.

Our next topic is to consider the cardinal add $(X)$, the additivity of the category for the space $X$. In order for $\operatorname{add}(X)$ to be defined we restrict ourselves to the case in which $\operatorname{cov}(X)$ is defined and $\operatorname{cov}(X)>\omega$. In such a case we put

$$
\operatorname{add}(X)=\inf \{|R|: R \text { is a family of nowhere dense subsets of } X
$$

$$
\text { and } \bigcup R \text { is not first category in } X\} \text {. }
$$

There is an obvious inequality: $\operatorname{add}(X) \leq \operatorname{cov}(X)$. Both cardinals $\operatorname{add}(X)$ and $\operatorname{cov}(X)$ have been intensively studied for $X$ being a metric separable space (see, for example, $[\mathbf{6}, \mathbf{7}])$. Many interesting and deep results have been obtained. One of the most celebrated results in this topic is the following inequality:

$(* *)$ If $\kappa$ is a cardinal such that $P(\kappa)$ holds and $X$ is a separable, metric second category space, then $\operatorname{add}(X)>\kappa$.

Here $P(\kappa)$ denotes the following combinatorial statement:

$P(\kappa)$ : If $S$ is a family of infinite subsets of $\omega$ such that $|S| \leq \kappa$, and any finite subfamily of $S$ has an infinite intersection, then there is an infinite subset $A$ of $\omega$ such that $A-s$ is finite for each $s \in S$.

The result $(* *)$ has been shown by D. Martin and R. Solovay [5]. We shall present a theorem that generalizes $(* *)$. Our generalization goes in two directions. First, it concerns a much wider class of spaces. Second, it uses a weaker assumption than $P(\kappa)$-namely, we shall use the following statement:

$P(\kappa) \downarrow$. If $\left\{T_{\alpha}: \alpha<\lambda\right\}$ is a family of infinite subsets of $\omega$ such that $\lambda \leq \kappa$ and $\alpha<\beta<\lambda$ implies $T_{\beta}-T_{\alpha}$ is finite, then there is an infinite subset $A$ of $\omega$ such that $A-T_{\alpha}$ is finite for each $\alpha<\lambda$.

Clearly, $P(\kappa) \downarrow$ follows from $P(\kappa)$. It is known [3] that $P\left(\omega_{1}\right)$ follows from $P\left(\omega_{1}\right) \downarrow$. However it is unknown whether $P(\kappa)$ is equivalent to $P(\kappa) \downarrow$, in general.

THEOREM 2. If $P(\kappa) \downarrow$ holds and $X$ is a second category space containing a dense countable subspace of points with countable character, then $\operatorname{add}(X)>\kappa$.

To prove this theorem we will need two lemmas. The first of these is already known; it goes back to F. Rothberger [10] though the explicit proof can be found in [3]. 


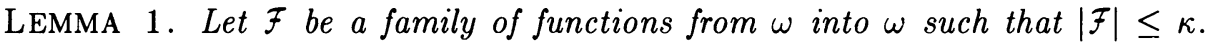
If $P(\kappa) \downarrow$ holds, then there is a function $g$ from $\omega$ into $\omega$ such that the set $\{n \in$ $\omega: g(n) \leq f(n)\}$ is finite for each $f \in \mathcal{F}$.

LEMMA 2. Let $X$ be a dense-in-itself $T_{1}$ space with a countable $\pi$-base, and let $\left\{D_{\alpha}: \alpha<\lambda\right\}$ be a family of countable dense subsets of $X$ such that $D_{\beta}-D_{\alpha}$ is finite whenever $\alpha<\beta<\lambda$. If $\lambda \leq \kappa$ and $P(\kappa) \downarrow$ holds, then there is dense subset $D$ of $X$ such that $D-D_{\alpha}$ is finite for each $\alpha<\lambda$.

Proof. Let $P$ be a countable $\pi$-base in $X$ consisting of nonempty open sets. For each $U \in P$ let us consider the family $\left\{U \cap D_{0} \cap D_{\alpha}: \alpha<\lambda\right\}$. Each such family consists of infinite subsets of the countable set $U \cap D_{0}$. This holds because $X$ is $T_{1}$ and the sets $D_{\alpha}$ are dense in $X$. We also have $U \cap D_{0} \cap D_{\beta}-U \cap D_{0} \cap D_{\alpha}$ is finite, whenever $\alpha<\beta<\lambda$. So we may apply $P(\kappa) \downarrow$, and we get that for any $U \in P$ there is an infinite subset $D_{U}$ of $U \cap D_{0}$ such that $D_{U}-D_{\alpha}$ is finite for each $\alpha<\lambda$. Enumerate $D_{U}$ by $\{d(U, n): n \in \omega\}$. Now for each $\alpha<\lambda$ define a function $f_{\alpha}: P \rightarrow \omega$, setting $f_{\alpha}(U)=\min \left\{n: d(U, m) \in D_{\alpha}\right.$ for each $\left.m \geq n\right\}$. Since $D_{U}-D_{\alpha}$ is finite for each $U \in \mathcal{P}$, the function $f_{\alpha}$ is well defined. We may apply our Lemma 1 to the family $\left\{f_{\alpha}: \alpha<\lambda\right\}$, and we get the existence of a function $g$ from $P$ into $\omega$ such that the set $\left\{U \in P: g(U) \leq f_{\alpha}(U)\right\}$ is finite for each $\alpha<\lambda$. Let us put $D=\{d(U, n): U \in P$ and $g(U) \leq n\}$. Then $D$ intersects each $D_{U}$ on an infinite set and therefore $D$ intersects each $U \in P$ on an infinite set. Since $P$ is a $\pi$-base in $X, D$ is dense in $X$. It remains to be shown that $D-D_{\alpha}$ is finite for each $\alpha<\lambda$. So fix $\alpha, \alpha<\lambda$. Let us observe that if $d(U, n) \in D-D_{\alpha}$, then $g(U) \leq f_{\alpha}(U)$ and $d(U, n) \in D_{U}-D_{\alpha}$. Hence $D-D_{\alpha} \subset \bigcup\left\{D_{U}-D_{\alpha}: U \in \mathcal{P}\right.$ and $\left.g(U) \leq f_{\alpha}(U)\right\}$. Because the sets $D_{U}-D_{\alpha}$ are finite and the set $\left\{U \in P: g(U) \leq f_{\alpha}(U)\right\}$ is finite as well, $D-D_{\alpha}$ is a finite set.

PROOF OF THEOREM 2. Assume, to the contrary, that there exists in the space $X$ a family $R$ consisting of nowhere dense subsets of $X$ such that $\bigcup R$ is second category in $X$ and yet $|R| \leq \kappa$. Without loss of generality we may assume that $X$ is dense-in-itself (otherwise apply the arguments below to the set int $\operatorname{cl} \bigcup R$ ). Let $D$ be a countable dense subset of $X$ consisting of points with countable character. For each $d \in D$ enumerate a countable base around $d$ by $\{U(d, n): n \in \omega\}$. We may also assume that $U(d, n) \subset U(d, m)$, whenever $m \leq n$. Of course, the family $\{U(d, n): d \in D$ and $n \in \omega\}$ forms a countable $\pi$-base in $X$. Knowing this we will be able to find a dense subset $C$ contained in $D$ such that $C \cap \operatorname{cl} F$ is finite for each $F \in R$. For this purpose enumerate the family $R$, say $R=\left\{F_{\alpha}: \alpha<\lambda\right\}$. Since $|R| \leq \kappa, \lambda \leq \kappa$. And now, we shall inductively define a family $\left\{D_{\alpha}: \alpha<\lambda\right\}$ satisfying the following conditions:

(i) $D_{\alpha} \subset D, D_{\alpha}$ is dense in $X$, and $D_{\alpha} \cap \operatorname{cl} F_{\alpha}=\varnothing$ for each $\alpha<\lambda$;

(ii) if $\alpha<\beta<\lambda$, then $D_{\beta}-D_{\alpha}$ is finite.

Put $D_{0}=D-\operatorname{cl} F_{0}$. Assume $\gamma<\lambda$ and $\left\{D_{\alpha}: \alpha<\gamma\right\}$ has already been defined. If $\gamma$ is a nonlimit ordinal, say $\gamma=\beta+1$, then it is enough to put $D_{\gamma}-D_{\beta}-\operatorname{cl} F_{\gamma}$. If $\gamma$ is a limit ordinal, then let $\left\{\alpha_{\xi} \xi<\operatorname{cf}(\gamma)\right\}$ be a strongly increasing sequence of ordinals less than $\gamma$. Because $\operatorname{cf}(\gamma)<\lambda \leq \kappa$ and $\operatorname{cf}(\gamma)$ is a cardinal, we may apply our Lemma 2 to the family $\left\{D_{\alpha_{\xi}}: \xi<\operatorname{cf}(\gamma)\right\}$. Hence we get a dense subset $\tilde{D}_{\gamma}$ of $X$ such that $\tilde{D}_{\gamma}-D_{\alpha_{\xi}}$ is finite for each $\xi<\operatorname{cf}(\gamma)$. We shall show that $\tilde{D}_{\gamma}-D_{\alpha}$ is finite for each $\alpha<\gamma$. So fix $\alpha, \alpha<\gamma$. There is $\xi, \xi<\operatorname{cf}(\gamma)$, such that $\alpha<\alpha_{\xi}$. 
Hence

$$
\tilde{D}_{\gamma}-D_{\alpha} \subset \tilde{D}_{\gamma}-\left(D_{\alpha} \cap D_{\alpha_{\xi}}\right) \subset\left(\tilde{D}_{\gamma}-D_{\alpha_{\xi}}\right) \cup\left(D_{\alpha_{\xi}}-D_{\alpha}\right)
$$

which is a finite set. Finally, if we put $D_{\gamma}=\left(\tilde{D}_{\gamma} \cap D\right)-\operatorname{cl} F_{\gamma}$ we get the desired set to complete the induction step.

Now, if we have a family $\left\{D_{\alpha}: \alpha<\lambda\right\}$ satisfying conditons (i) and (ii), we shall again apply our Lemma 2 to this family (since $\lambda \leq \kappa)$, and we get a dense subset $C$ of $X$ such that $C-D_{\alpha}$ is finite for each $\alpha<\lambda$. We may assume that $C$ is a subset of $D$ (throwing out finitely many points if needed); the properties $C-D_{\alpha}$ is finite and $D_{\alpha} \cap \operatorname{cl} F_{\alpha}=\varnothing$ give us that $C \cap \operatorname{cl} F_{\alpha}$ is finite, so $C \cap \operatorname{cl} F$ is finite for each $F \in R$.

Enumerate the points of $C$ by $\left\{c_{n}: n \in \omega\right\}$ and consider the families $R_{n}=\{F \in$ $\left.R: C \cap \operatorname{cl} F \subset\left\{c_{0}, \ldots, c_{n}\right\}\right\}$. Each member of $R$ falls into one of the families $R_{n}$. Hence one of them, say $R_{m}$, has the union of its members being second category. Let us throw out $m+1$ first points from the set $C$. Then we obtain a dense countable set $B$ such that $B \cap \operatorname{cl} F=\varnothing$ for each $F \in R_{m}$. Now for each $F \in R_{m}$ define a function $f_{F}: B \rightarrow \omega$ setting $f_{F}(b)=\min \{n: U(b, n) \cap \operatorname{cl} F=\varnothing\}$ (recall that $B$ is a subset of $D$ and for each $d \in D$ we have assigned a local base, $\{U(d, n): n \in \omega\})$. Since $b \notin \operatorname{cl} F$ for each $F \in R_{m}$, the function $f_{F}$ is well defined. We may apply our Lemma 1 to the family $\left\{f_{F}: F \in R_{m}\right\}$, and we get the existence of a function $g: B \rightarrow \omega$ such that the set $\left\{b \in B: g(b) \leq f_{F}(b)\right\}$ is finite for each $F \in R_{m}$. For each finite subset $s$ of the set $B$ let us put $E_{s}=X-\bigcup\{U(b, g(b)): b \in B-s\}$. The number of the sets $E_{s}$ is countable, since $B$ is a countable set. So we will come to a contradiction if we show that $\bigcup R_{m} \subset \bigcup\left\{E_{s}: S\right.$ is a finite subset of $\left.B\right\}$ and that each set $E_{s}$ is nowhere dense in $X$.

To see that $\bigcup R_{m} \subset\left\{E_{s}: s\right.$ is a finite subset of $\left.B\right\}$, let us observe that $F \subset E_{s}$, whenever $s=\left\{b \in B: g(b) \leq f_{F}(b)\right\}$.

To see that $E_{s}$ is nowhere dense, take an arbitrary nonempty open subset $V$ of $X$. The $V \cap B$ is infinite and therefore there is a point $b_{0}$ belonging to $V \cap B-s$. Consequently,

$$
b_{0} \in V \cap U\left(b_{0}, g\left(b_{0}\right)\right) \subset V \cap \bigcup\{U(b, g(b)): b \in B-s\} .
$$

Our final topic is to consider the cardinal $u(X)$. It is defined (if possible) in the following way:

$$
u(X)=\inf \{|Y|: Y \text { is a second category subset of } X\} .
$$

There are obvious inequalities between $\operatorname{add}(X)$ and both cardinals $\operatorname{cov}(X)$ and $u(X)$ in the case in which $\operatorname{cov}(X)$ is defined and $\operatorname{cov}(X)>\omega$, namely $\operatorname{add}(X) \leq u(X)$ and $\operatorname{add}(X) \leq \operatorname{cov}(X)$. Within ZFC one cannot prove the equalities add $(X)=u(X)$ or $u(X)=\operatorname{cov}(X)$, even if $X$ is the space of reals [6].

$M(\kappa)$. If $\mathcal{F}$ is a family of functions from $\omega$ into $\omega$ such that $|\mathcal{F}| \leq \kappa$, then there is a function $g$ from $\omega$ into $\omega$ such that the set $\{n \in \omega: g(n)=f(n)\}$ is finite for each $f \in \mathcal{F}$.

THEOREM 3. If $M(\kappa)$ holds and $X$ is a dense-in-itself second category Hausdorff space with a countable $\pi$-base, then $u(X)>\kappa$.

ProOF. Assume, to the contrary, that the space $X$ contains a second category subset $Y$ such that $|Y| \leq \kappa$. Let $\mathcal{P}$ be a countable $\pi$-base in $X$. Because $X$ is a 
dense-in-itself Hausdorff space, each nonempty open subset of $X$ contains infinitely many disjoint nonempty open subsets. For any $u \in P, u \neq \varnothing$, let $\{u(n): n \in \omega\}$ be a disjoint family of nonempty open subsets of the set $u$. Now, for any $y \in Y$ define a function $f_{y}: P-\{\varnothing\} \rightarrow \omega$ setting $f_{y}(u)=n$ if $y \in u(n)$ and $f_{y}(u)=0$ if $y \notin \bigcup\{u(n): n \in \omega\}$. Because $|Y| \leq \kappa$ and $M(\kappa)$ holds, there is a function $g$ from $P-\{\varnothing\}$ into $\omega$ such that the set $\left\{u \in P-\{\varnothing\}: g(u)=f_{y}(u)\right\}$ is finite for each $y \in Y$. For each finite subset $s$ of the set $P$ let us put $E_{s}=X-\bigcup\{u(g(u)): u \in P-s\}$. The number of the sets $E_{s}$ is countable. So, we will come to a contradiction if we show that $Y \subset \bigcup\left\{E_{s}: s\right.$ is a finite subset of $\left.P\right\}$ and that each set $E_{s}$ is nowhere dense. To see the first observe that if $y \in Y$, then $y \in E_{s}$, where $s=\{u \in$ $\left.P-\{\varnothing\}: g(u)=f_{y}(u)\right\}$. To see that each $E_{s}$ is nowhere dense, fix $s$ and take an arbitrary nonempty open subset $V$ of $X$. Because $X$ is dense in itself, the set of all members of $P$ contained in $V$ is infinite, and therefore there is one in $P-(s \cup\{\varnothing\})$. Hence $V \cap \bigcup\{u(g(u)): u \in P-s\} \neq \varnothing$.

In [6, Theorem 1.3] some analogous results have been obtained for metric separable spaces.

\section{REFERENCES}

1. N. Bourbaki, Topologie générale, Chapitre 9, Hermann, Paris, 1958.

2. J. Dobðs, A note on the invariance of Baire spaces under mappings, Časopis Pěst. Mat. 108 (1983), 409-411.

3. D. Fremlin, Consequences of Martin's Axiom, Cambridge Univ. Press, 1984.

4. Z. Frolik, Remarks concerning the invariance of Baire spaces under mappings, Czechoslavak Math. J. 81 (1961), 381-385.

5. D. A. Martin and R. M. Solovay, Internal Cohen extensions, Ann. Math. Logic 2 (1970), 143-178.

6. A. W. Miller, Some properties of measure and category, Trans. Amer. Math. Soc. 266 (1981), 93-114.

7. _ A characterization of the least cardinal for which the Baire category fails, Proc. Amer. Math. Soc. 86 (1982), 498-502.

8. T. Neubrunn, A note on mappings of Baire spaces, Math. Slovaca 27 (1977), 173-176.

9. _ Erratum, Math. Slovaca 27 (1977), 442.

10. F. Rothberger, On some problems of Hausdorff and of Sierpinski, Fund. Math. 35 (1948), 29-46.

Department of MAThematical and Computer SCiences, Youngstown State UNIVERSITY, YOUNGSTOWN, OHIO 44555 (Current address of Zibigniew Piotrowski)

Current address (Andrzej Szymański): Department of Mathematics, Slippery Rock University, Slippery Rock, Pennsylvania 16057 